Chirurg 2017 $\cdot 88: 802$

DOI 10.1007/s00104-017-0485-1

Online publiziert: 27. Juli 2017

๑) Springer Medizin Verlag GmbH 2017

CrossMark

M. Schrempf $\cdot$ M. Anthuber

Klinik für Allgemein-, Viszeral- Transplantationschirurgie, Klinikum Augsburg, Augsburg, Deutschland

\title{
Stent vs. Stoma bei maligner Kolonstenose - Langzeitergebnisse
}

häufiger in High-volume-Zentren als in Medium- und Low-volume-Zentren behandelt. Ein verlängerter Krankenhausaufenthalt war in der Stent-Gruppe seltener als in der Stoma-Gruppe (Odds Ratio $[\mathrm{OR}]=0,50$; $95 \%$-Konfidenzintervall [KI] 0,26-0,97; $p=0,04)$. Die Komplikationsrate während des stationären Aufenthalts und die Rate an Wiederaufnahmen innerhalb von 90 Tagen und innerhalb eines Jahres unterschieden sich nicht zwischen den beiden Gruppen. Patienten in der Stent-Gruppe wurden signifikant seltener in Pflegeund Rehaeinrichtungen entlassen und konnten häufiger wieder in ihr häusliches Umfeld zurückkehren als Patienten der Stoma-Gruppe (OR = 0,14; $95 \%$-KI 0,07-0,28; $p<0,01)$. Die Perforationsrate in der Stent-Gruppe lag unter $6 \%$. In der Stent-Gruppe erfolgten innerhalb eines Jahres mehr Zweiteingriffe als in der Stoma-Gruppe (OR = 2,93; $95 \%$ $\mathrm{KI}=1,12-7,68 ; p=0,03)$. Über $70 \%$ dieser Zweiteingriffe bestanden in einer erneuten Stenteinlage.

Diskussion und Fazit. Die Ergebnisse dieser Arbeit zeigen, dass die palliative Stenteinlage bei maligner Dickdarmstenose eine sinnvolle Alternative zur Stomaanlage darstellt. Im Vergleich mit anderen Studien war die Perforationsrate in der vorgestellten Arbeit gering [1]. Dies ist möglicherweise auf die Expertise in den High-volume-Zentren zurückzuführen, in denen ein Großteil der Stenteinlagen erfolgte. Der wesentliche Vorteil der Stenteinlage bestand in der Vermeidung eines verlängerten Krankenhausaufenthalts, der häufigeren Entlassung in das häusliche Umfeld und in der Vermeidung einer Stomaanlage. Diese Faktoren können in der Palliativsituation die Lebensqualität der betroffenen Patienten erheblich steigern. Andererseits kann ein Stoma die Pflege bettlägeriger Patienten vereinfachen. Bei der Wahl des Therapiekonzepts sollte zudem berücksichtigt werden, dass für Patienten unter Therapie mit Bevacizumab ein erhöhtes Perforationsrisiko im Rahmen der Stenteinlage beschrieben wurde [2].

\section{Korrespondenzadresse}

Prof. Dr. M. Anthuber

Klinik für Allgemein-, Viszeral- Transplantationschirurgie, Klinikum Augsburg

Stenglinstr. 2, 86156 Augsburg, Deutschland matthias.anthuber@klinikum-augsburg.de

Interessenkonflikt. M. Schrempf und M. Anthuber geben an, dass kein Interessenkonflikt besteht.

\section{Literatur}

1. van Hooft JE, Bemelman WA, Oldenburg $B$ et al (2011) Colonic stenting versus emergency surgery for acute left-sided malignant colonic obstruction a multicentre randomised trial. Lancet Oncol 12:344-352

2. Small AJ, Coelho-Prabhu N, Baron TH (2010) Endoscopic placement of self-expandable metal stents for malignant colonic obstruction: long-term outcomes and complication factors. Gastrointest Endosc 71:560-572 